\title{
Real space frequency analysis of electron diffuse scattering for lattice distortion determination in real crystals
}

\author{
Jian-Min Zuo, Yu-Tsun Shao, Haw-Wen Hsiao, Renliang Yuan, Qun Yang, Yang Hu
}

University of Illinois at Urbana-Champaign, Urbana, United States of America;

\author{
jianzuo@illinois.edu
}

One of major crystallographic challenges is how to determine the structure of severely distorted crystal lattices, such as at a dislocation core and in high entropy alloys where distortion is non-uniform. Here we propose a new 4D scanning transmission electron microscopy (4D-STEM) based technique, called Cepstral STEM, for imaging disordered crystals using electron diffuse scattering. Local fluctuations of diffuse scattering are captured by scanning electron nanodiffraction (SEND) using a coherent probe. The harmonic signals in electron diffuse scattering are detected through Cepstral analysis and used for imaging. By integrating Cepstral analysis with 4D-STEM, we demonstrate that information about the distortive part of electron scattering potential can be separated and imaged at $\mathrm{nm}$ spatial resolution. We apply our technique to the analysis of a dislocation core in SiGe and lattice distortions in high entropy alloy [1].

[1] Yu-Tsun Shao, Renliang Yuan, Haw-Wen Hsiao, Qun Yang, Yang Hu, and Jian-Min Zuo, "Cepstral scanning transmission electron microscopy imaging of severe lattice distortions", Ultramicroscopy, 113252 (2021).

Keywords: Lattice distortion, diffuse scattering, high entropy alloys 